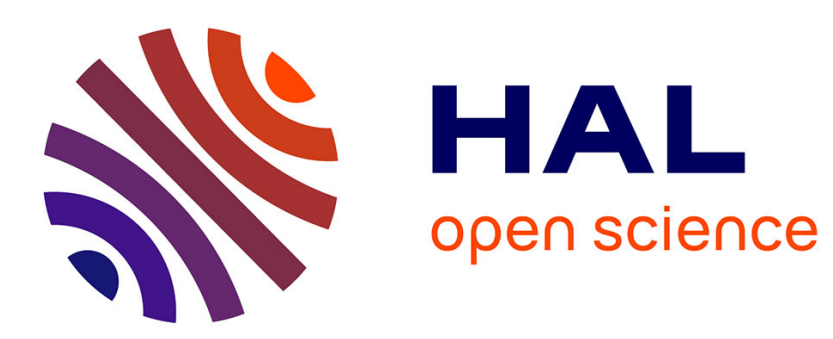

\title{
Neural network applications to reservoirs: Physics-based models and data models
}

\author{
Tahar Aifa
}

\section{To cite this version:}

Tahar Aifa. Neural network applications to reservoirs: Physics-based models and data models. Journal of Petroleum Science and Engineering, 2014, insu-01084932, 123, pp.1-6. 10.1016/j.petrol.2014.10.015 . insu-01084932

\section{HAL Id: insu-01084932 \\ https://hal-insu.archives-ouvertes.fr/insu-01084932}

Submitted on 20 Nov 2014

HAL is a multi-disciplinary open access archive for the deposit and dissemination of scientific research documents, whether they are published or not. The documents may come from teaching and research institutions in France or abroad, or from public or private research centers.
L'archive ouverte pluridisciplinaire HAL, est destinée au dépôt et à la diffusion de documents scientifiques de niveau recherche, publiés ou non, émanant des établissements d'enseignement et de recherche français ou étrangers, des laboratoires publics ou privés. 


\section{Editorial}

\section{Neural network applications to reservoirs: Physics-based models and data models}

\section{Introduction}

Neural computations such as artificial neural networks (ANN) have aroused considerable interest over the last decades (e.g. Nikravesh et al., 2003; Graupe, 2007), and are being successfully applied across a wide range of problem areas, to domains as diverse as medicine, finance, engineering, geology and physics, to problems of complex dynamics and complex behaviour prediction, classification or control. Several architectures, learning strategies and algorithms have been introduced into this highly dynamic field (e.g. Nikravesh et al., 2003; Sandham and Leggett, 2003; Aminzadeh and de Groot, 2006 ; Adeniran et al., 2010). Such new tools for the investigation of reservoirs are evaluated and tested during drilling processes and through logging analyses. This special volume is dedicated to the use of artificial intelligence in reservoir investigations, where physics-based models and data models are the core of the volume. Prediction of petrophysical parameters through various modern tools and technologies based on computational and analytical procedures (theory and applications) are also presented.

This project, which has been carried out along with a collection of twenty out of fifty research papers selected for this special volume, aims to better define and configure various kinds of reservoirs, and to predict their characteristics. Comparisons of reservoirs and methodologies for the checking of their characteristics are very highly appreciated and considered by the petroleum industry, especially when data on some petrophysical parameters were lacking to fix the physical models and track their limitations. The challenge between data models and their relationship with physics-based models have also been considered. Numerous research works among which some related books (e.g. Jerry Lucia, 2007; Ashton Acton, 2013; Shi, 2014) were published to show the importance of investigating reservoirs to further optimize production. Different techniques to forecast parameters can be used but every methodology has its own limitations, either in terms of accuracy or of time processing. We are mainly dealing with non-linear inverse problems which can be rather complex. How can we control the convergence and its speed? How can we avoid local minima? What is the best solution for a specific reservoir? Can we adapt some techniques to cases where petrophysical parameters can record quick, sensitive variations ? How can we manage to meet this challenge, the choice of techniques in the case of a specific reservoir, so as to maximize hydrocarbon recovery, while minimizing the expenditure of time and money? It is sometimes a dilemma to choose a technique which itself has limiting areas of expertise or output characteristics sometimes not fully adapted to the studied case, especially when dealing with heterogeneous complex systems.

Most of the topics summarized below were discussed within this volume through the suggested selected papers: 
- Analysis of synthetic and real petrophysical data of reservoirs: data models vs. physical models

- Genetic algorithms combined to ANN applied to reservoir simulation and recognition

- Neural network, algorithms, softwares and their applications to reservoirs

- Inversion techniques in reservoir complex systems compared to ANN and their limitations

- Prediction of petrophysical parameters in reservoirs

- ANN to simulate reservoirs for EOR prediction

- Tight gas reservoir development using ANN

- Prediction of natural and induced fracturation at various scales: theory and applications

- Fluids vs. mineralizations and fracture zones and their impacts on modelling

- ANN and 3D modelling of heterogeneous reservoirs including unconventional reservoirs

This volume includes suitable papers for scientists, engineers and practitioners interested in the study, analysis, modelling and implementation of neural computing systems, both theoretically and in a broad range of applications to oil and gas conventional or unconventional reservoirs. The papers we present highlight the main ideas to better investigate reservoirs and their characteristics for further applications (fracturation, simulation, EOR, etc.). The various contributions we have compiled collectively provide an integrated and useful perspective for those who are involved in petroleum exploration research or are looking for new tools of investigation to characterize reservoirs and/or to develop new research themes or tools of prediction of related parameters based on artificial intelligence.

\section{Novelties in ANN reservoir predictions: case studies}

\section{(i) A new way of investigation of gas condensate reservoirs}

Ahmadi et al. (2014a) developed a robust, ingenious tool to estimate and monitor the dew point pressure $\left(\mathrm{P}_{\mathrm{d}}\right)$ of gas condensate reservoirs. They show that the hybrid method they presented, i.e. Particule Swarm Optimisation - Artificial Neural Network (PSO-ANN), composed of swarm intelligence and ANN can record and help to understand reservoir fluid behaviour within different simulation cases. They based their research on the use of accurate, experimental $\mathrm{P}_{\mathrm{d}}$ data reported in previous surveys, introduced into the proposed model to tune and validated the suggested approach through retrograded gas condensate reservoirs. The results show that (i) an acceptable similarity between $\mathrm{P}_{\mathrm{d}}$ obtained from the PSO-ANN model compared with the relevant actual $\mathrm{P}_{d}$ values, (ii) this smart, cheap and user-friendly model, can become a substitute when essential $P_{d}$ data are unavailable.

An ANN method based on well test data to identify gas condensate reservoirs has been used by Ghaffarian et al. (2014). As we may know, gas condensate reservoirs are more complex than oil and dry gas reservoirs. The methodology they presented enabled the detection of a reservoir model and its boundaries performed through trial-and-error procedures. As the governing partial derivative equation related to gas condensate well testing operation is non-linear, it has been converted to pseudo-pressure for its linearization. Various models of gas condensate reservoirs have been identified through applying the ANN approach on the pseudo-pressure derivative plots. A single multi-layer perceptron (MLP) neural network has been used to recognize twelve different reservoir-boundary models. Then 
by clustering the reservoir models into three clusters based on the similarities of their pattern, three different MLP networks have been designed to improve the capability of single MLP for gas condensate model identification. The data points of pseudo-pressure derivative plots are introduced to the network as an input pattern. The required training, testing and validating datasets are generated using professional well test software (PANSYS) and show that 3-MLP clustering model is better than a single one.

\section{(ii) History matching}

Many applications in uncertainty analyses can be reached through ANN, but only a few of them are addressed in history matching processes for reservoir characterization. History matching processes were used by Costa et al. (2014) through an application of ANN to simulate production strategies and to perform forecasts. They were able (i) to evaluate the application of proxy models generated through ANN tools, and (ii) to analyze the influence of a training dataset in the ANN performance. They also show that sampling technique is important to the process to be trained by feed forward network. To optimize the process, other techniques can also be tested, for instance a genetic algorithm (GA) or particule swarm optimisation (PSO) to find the global minimum and thus a suitable configuration for the studied case.

Foroud et al. (2014) used ANN to assist history matching based on geomodelling of Brugge field and a fractured Iranian reservoir. The quantification of sub-surface uncertainties at the origin of reservoir simulation is a crucial problem, especially when dealing with a field under investigation or development. In this case, history matching may minimize the difference between simulation results and observed field data, but the solution is not unique and can be a challenge for optimization. ANN may help to bring a possible option to improve the quality of the studied field. This has been demonstrated through this paper where the time-consuming uncertainties are drastically reduced.

In this section, we may also notice that Maschio and Schiozer (2014) introduced a Bayesian history matching using ANN and Markov Chain Monte Carlo (MCMC) to solve some inverse problems by sampling techniques, i.e. the Metropolis-Hastings ( $\mathrm{MH}$ ) algorithm which was suitable to sample the posterior distribution but with some computational cost to converge. Both the combination of MCMC and an adequately trained ANN helped to solve the realistic 16 uncertain attributes for the suggested reservoir model with uncertainty reduction. The iteration procedure developed here induced a significant reduction of the computational time.

\section{(iii) Net pay determination - oil or gas recovery}

In the same manner, Masoudi et al. (2014a) used ANN to determine net pay zones within two Iranian offshore oil fields: a carbonate reservoir (Mishrif), and a sandy or clastic one (Burgan). It plainly appears in both geological environments that the application of ANN technique showed results nearly similar to well test results. It could be trained by raw well-logs, independently from laboratory reports 
of porosity, permeability and shale content; but could also provide fuzzified output compatible with heterogeneous reservoirs such as carbonates.

In the second case, Masoudi et al. (2014b) applied the Dempster-Shafer rule of combination to net pay detection to better estimate, model, simulate and plan production reservoirs. The Dempster-Shafer theory (DST), which is a generalization of the Bayesian theory (conditional probabilities), has been carefully applied to previous reservoirs using porosity, shale volume and water saturation. The results are compared to well tests and to the output of the conventional cut-off based method. The conventional cut-off based method, using petrophysical cut-offs on well-logs often used in sandy reservoirs, works nicely but hardly or rarely provides suitable results in carbonated reservoirs. They show that DST, which does not need to be trained, provides a continuous fuzzy output, compatible with geological features and well-test data.

The increase of gas production worldwide is induced by the demand for natural gas, so multi-lateral well techniques were developed to improve the production techniques. Supervised training algorithms are often used in the oil and gas industry because of their accuracy in solving many challenging and complex problems. The paper of Enab and Ertekin (2014) presents an ANN tool able to evaluate the dual horizontal well in a tight gas reservoir. The predicted data include flow rate and gas recovery profiles, dual horizontal well configuration and pattern size. For that purpose, networks, forward and inverse, were developed. Monte Carlo simulation was implemented in the resulting tool to guide the user while specifying the reservoir ultimate goal. The ANNs were trained, validated and tested using the training data generated by a commercial simulator. The developed forward and inverse tools were tested and gave a mean square error of $7.5 \%$ and $9.8 \%$, respectively. They are capable of comparing thousands of different input combinations much more rapidly than a commercial simulator. A total of 1250 random combinations of the reservoir properties and dual horizontal well configuration parameters, within the selected limits, have been generated to predict the gas recovery profiles using a commercial reservoir simulator. The forward ANN was developed to predict the flow rate and gas recovery profiles for chosen dual horizontal well configuration and pattern size over a specified production period (50 years). The inverse ANN was developed to predict the dual horizontal well configuration and pattern size that can be used to fulfil the desired gas recovery over the same production period. Finally, the utilization of the multi-lateral well techniques helped to optimize the cumulative fluid recovery, and to minimize the environmental impacts, and the drilling and completion costs.

The work presented by Mosobalaje et al. (2014) requires two techniques for estimating permeability and well drainage radius, for solution-gas driven reservoirs. However, data requirement has placed a limitation on the application of the techniques. Applying such techniques requires readily-available production data cumulative production and production rate vs. time. In addition, it also requires the scarcely-measured average reservoir pressure $(\overline{\mathrm{P}})$, and average oil saturation $\left(\overline{\mathrm{S}_{0}}\right)$ vs. time. This work presents a practical method for deriving the scarcely-measured data from the readily-available data. 
This method, based on a new solution methodology to the material balance equation (MBE) for solution-gas driven reservoirs, is presented as a subroutine, added to the procedures of the property estimation techniques. It is analytically based on the equality of the fluid withdrawal terms (left hand side, LHS) and fluid expansion terms (right hand side, RHS) of the conventional MBE, and the pressure value that upholds the equality. This means that the difference between LHS and RHS terms must be approximately zero.

The authors applied this subroutine to two reservoir models yielding excellent estimates of $\overline{\mathrm{P}}$ and $\overline{\mathrm{S}_{0}}$ data and exhibiting good agreement with $\overline{\mathrm{P}}$ and $\overline{\mathrm{S}_{0}}$ data resulting from simulating the reservoirs. Furthermore, this subroutine generated $\overline{\mathrm{P}}$ and $\overline{\mathrm{S}_{0}}$ data that have been used in implementing the property estimation techniques. The results obtained, i.e. permeability, well drainage radius, match well both the results of the techniques' implementation using simulator's $\bar{P}$ and $\overline{\mathrm{S}_{0}}$ data, and with the true values of these properties.

\section{(iv) Fracturation - Impact on parameters and production optimization}

Sheremetov et al. (2014) discussed the results of modelling naturally fractured reservoirs based on the application of the non-linear autoregressive neural network with exogenous inputs (NARX). The NARX architecture, based on time-series (TS) forecasting, was used in 42 wells of the JujoTecominoacán oilfield (Gulf of Mexico) with a highly heterogeneous system. This technique can be applied properly to multivariate multi-step ahead prediction of reservoir dynamics using the Hurst exponent. Real data for forecasting parameters through 31 input variables related to (a) oil, gas and water productions, (b) flowing bottom-pressure, (c) choke sizes, (d) completion interval depth, and (e) cleaning and stimulation treatments were used in the experiments. Beside oil production, modelling the behaviour of 3 dynamic variables such as gas, water and bottom-hole pressure (BHP) are settled as output variables. In this paper ANNs were addressed to TS analysis and to the prediction of reservoir parameters (PVT, viscosity, permeability, porosity, etc.). The NARX model depends on several parameters (embedded memory of input and output, number of neurons in the input and hidden layers, activation function, etc.). An attempt to predict dynamic fractures was elaborated through several solutions in this paper.

Aïfa et al. (2014a) used a new tool to investigate fracture zones within reservoirs. It is based on magnetic susceptibility (Ms) measurements along the cores of 6 wells. This time-saving, nondestructive technique has been applied to the Hamra quartzites reservoir (Hassi Messaoud oilfield), composed mainly of quartz and is poorly mineralized, except in some fractured areas. But it could be applied successfully to carbonate reservoirs as well, since the contrasts in Ms coincide with high fracture density and high shale content (increase of gamma ray in this interval). The application of Principal Component Analysis (PCA) to the entire reservoir shows low linear correlation between Ms and the main petrophysical parameters (gamma ray, neutron porosity, density, and oil saturation). 
Meanwhile, the application of fuzzy ranking and ANN evidenced non-linear relations between these parameters. It is justified by the prediction of the Ms from the petrophysical parameters with an acceptable degree of accuracy. The results obtained using an ANN structure of 25 neurons in a hidden layer show the performance in the test stage with low mean square error (MSE) and mean relative error (MRE), and high correlation coefficient (R). The knowledge of such Ms behaviour within the reservoir can be a reliable complementary indicator to locate fracture density for further hydraulic fracturing to produce oil in tight sand reservoirs. The complexity of fracturing phenomena needs to be mastered by several magnetic factors such as Ms, magnetic intensity and direction of the natural remanent magnetization (NRM), possibly combined with CBIL and UBI tools to orientate the fractures. The prediction of Ms from petrophysical parameters using ANN indicated a good performance in the training and generalization phase, confirming a non-linear relation between parameters.

To select well refracture candidates Yanfang and Salehi (2014) used a hybrid simulation by merging mathematical data analysis with feed forward BP-ANN on post-fracturing data. A model preference, based on the correlation coefficients of several selected independent variables against production performance, was chosen. The solution to this expense is a tool able to identify restimulation candidates quickly and economically. For this reason, they applied two mathematical analysis techniques to filter several independent yet influential parameters as inputs, supposed to be primary factors with a high impact on potential production improvement. Then they train and predict postfracture production with these well data. The errors of the best samples should decrease consistently along with the training samples. A minimal error of the training sets is not necessary because overfitting of the network could be memorizing rather than generalizing. The testing results show that there is higher than $80 \%$ prediction accuracy, which is good enough for decision making. This methodology was applied in Zhongyuan oilfield (central China) which belongs to a low permeability reservoir with very complicated petrophysical properties and gave credible prediction results.

\section{(v) Sand production - Porosity/Permeability forecasting}

Porosity is one of the most important parameters of the hydrocarbon reservoirs, the accurate knowledge of which allows petroleum engineers to have adequate tools to evaluate and minimize the risk and uncertainty in the exploration and production of oil and gas reservoirs. Different direct and indirect methods are used to measure this parameter, most of which (e.g. core analysis) are time and cost-consuming. Hence, applying an efficient method that can model porosity is important. We may show that the capability (i.e. classification, pattern matching, optimization and data mining) of an ANN is suitable for inherent uncertainties and imperfections found in petroleum engineering problems considering its successful application. Moreover, other restrictions, especially when cores are not available or are lacking in certain intervals, lead to the application of other methods to predict porosity. Furthermore, most of the available log tools cannot directly measure the porosity and one has 
to interpret them. Thus, to find a relationship between the widely available parameters in reservoir and porosity (e.g. sonic transit time and density logs) to obtain the porosity indirectly sometimes becomes a crucial problem. Conversion formulas usually contain terms and factors that respectively depend on the individual location and lithology (e.g. clay content, pore fluid type, grain density and grain transit time for the conversion from sonic transit time and density logs). These equations, however, are often unreliable.

Another problem with water flow into hydrocarbon bearing may occur and cause changes in relative permeability or weakening of the overall strength of the rock. Chemical interactions may also occur between water and rock cementation and affect the sand production.

To this aim, using a method that enables predicting the porosity and/or permeability in different heterogeneity conditions of reservoir becomes necessary.

Khamehchi et al. (2014) applied a novel approach to sand production using ANN. Critical total drawdown (CTD) as an index of sand production onset in oil and gas wells was aimed to be estimated through 4 proposed methods: (i) multiple linear regression (MLR), (ii) MLR in which coefficients are optimized using a genetic algorithm (GA-MLR), (iii) a back propagation neural network (BP-ANN), and (iv) a particle swarm optimisation neural network (PSO-ANN). They used 23 field datasets collected from problematic wells of the North Adriatic Sea to develop and predict these models. They show that the performance of PSO-ANN is better than BP-ANN in the training, testing and all phases. Results from the simple regression analysis showed that there are statistically meaningful relationships between CTD with total vertical depth, effective over-burden vertical stress, transit time and cohesive strength. MLR and GA evolved MLR models have been presented to correlate the CTD to the four statistically important parameters. The results show that GA can just slightly improve the accuracy of the MLR model. Two neural network models with the algorithms of BP-ANN and PSO-ANN have been constructed to detect the complex relationships between the CTD and all affecting parameters extracted from the literature. Both networks with one hidden layer showed the best performance among different structures. Such results illustrate that the predictive performance of both BP-ANN and PSO-ANN models are much better than the traditional MLR and GA evolved MLR. However, PSO showed to be more effective to optimize the weights of the neural network.

The paper of Chaki et al. (2014) proposes a modular ANN (MANN) concept to predict sand fraction from seismic attributes (seismic impedance, instantaneous amplitude, and instantaneous frequency). It is in fact based on pre-processing, modelling, and post-processing stages to carry out well top guided prediction. In the process of mapping sand fraction from seismic attributes, the authors extracted the first seismic attributes from a 3D seismic cube at eight well locations of a western onshore hydrocarbon field, India. Then, integration of seismic and borehole data are carried out using timedepth relationship information at the available well locations. The pre-processed master dataset is then divided into three zones based on the two well tops such as the first available patterns to top 1, top 1 to top 2, and top 2 to the last available data pattern. In the model building and validation stage, three 
networks have been designed for three different zones separately. Sand fraction and three seismic attributes corresponding to seven wells were used for training and testing, and the patterns corresponding to the remaining well were used for blind prediction. The satisfactory performance in blind testing encourages to carry out volumetric prediction of sand fraction using the three trained models. The results evaluated by three different networks (zone-wise) are merged to form a volumetric cube containing the estimated sand fraction values across the study area along the entire depth range. The application of multiple simpler networks instead of a single one improves the prediction accuracy in terms of performance evaluators-correlation coefficient, root mean square error, absolute mean error and program execution time. Then, a model building and validation, based on volumetric prediction of reservoir properties, is carried out using calibrated network parameters. This stage is followed by postprocessing to improve visualization. Thus, a complete framework, which includes pre-processing, model building and validation, volumetric prediction, and post-processing, is designed for successful mapping between seismic attributes and a reservoir characteristic. The proposed framework outperformed a single ANN in terms of reduced prediction error, program execution time and improved correlation coefficient as a result of application of the MANN concept.

Rabbani et al. (2014) developed a new method for extraction of pore network from 3-D realistic micro-tomography images. They used these 3-D scanned data to analyze and extract pore network, assuming pores as spheres and throats as cylinders, through the watershed segmentation algorithm (WSA). In this paper, a new simple method is developed to detect pores and throats for analyzing the connectivity and permeability of the network. This automated method uses some of the common and well-known image processing functions which are widely accessible by researchers and this has led to an easy algorithm implementation. In this method, after polishing and quality control of images, using city-block distance function and WSA, pores and throats are detected and 3-D network is produced. This method can also be applied on 2-D images of sedimentary rocks such as thin sections of rock and other porous media to extract some characteristics of the porous media for instance pore and throat size distribution. The results of network extraction were verified by comparing the distribution of the coordination number with a prevalent method: the maximal ball.

In their paper, Shokooh Saljooghi and Hezarkhani (2014) introduced an alternative method of porosity prediction, based on integration between wavelet theory and ANN called wavelet neural network (WNN) or wavenet. It can be used for function approximation to static and dynamic non-linear inputoutput modelling of processes. The wavenets use wavelet functions as hidden neuron activation functions which can be applied to predict porosity from well log data. The efficiency of this type of network in function learning and estimation is compared to that of ANNs. The simulation results indicate decrease in estimation error values that depicts its ability to enhance the function approximation capability, and consequently exhibit excellent learning ability compared to the conventional ANN with sigmoid or other activation functions. The wavenet improves the performance of the trained network for fast convergence, resistance to noise interference, and high complex ability 
to learn and track unknown/undefined complex systems. In practice, different wavelets (Mexican hat, Morlet, Shannon, etc.) were applied to real well log data to predict porosity. The results show that the WNN, with $94 \%$ correlation coefficient for porosity prediction, would be an appropriate substitute for ANN with $89 \%$ correlation coefficient. The wavenet provides a pragmatic solution to the problem of converting well $\operatorname{logs}$ to reservoir properties. Numerical results indicate that substituting different wavelet functions as feed forward neural network transfer functions can enhance the network performance and efficiency.

Ahmadi and Ebadi (2014) applied a new approach based on ANN concept to monitor permeability and porosity of petroleum reservoirs by means of petrophysical logs in various conditions. To tackle referred issue, they carried out different artificial intelligence techniques including fuzzy logic (FL) optimized by GA and least square support vector machine (LSSVM). The intelligent approaches developed are examined by implementing extensive real field data from northern Persian Gulf oil fields. The results obtained from the intelligent approaches developed are compared with the corresponding real petrophysical data and gained outcomes of the other conventional models. The correlation coefficient between the model estimations and the relevant actual data is found to be greater than 0.96 for GA-FL approach and 0.97 for GA-LSSVM. The results indicate that implication of GA-LSSVM and GA-FL in prediction can lead to more reliable porosity/permeability predictions, thus designing more efficient reservoir simulation schemes. They also observed that the MSE was set as the fitness function in the first run but in the next run, $\mathrm{R}^{2}$ played this role of the fitness function. A simple statistical comparison between both groups of responses clarified the superiority of choosing $\mathrm{R}^{2}$ as the fitness function which had resulted in producing outputs closer to real ones than setting the MSE. Running the GA optimization reduces the percentages of the relative error groups with high rates. The LSSVM approach has the potential of preventing being trapped in local optimal for estimation of permeability/porosity, since this new model has both local and global search engines. This approach for estimation of permeability/porosity of the reservoir, determination of the optimal value of the two main parameters such as regularization $\gamma$ and RBF kernel $\sigma^{2}$ present a big challenge which requires further research work.

\section{Genetic algorithms : What for?}

The genetic algorithms are a usual paradigm for the optimization of system analysis. In the case of the training, analytical solutions such as the gradient descents are often preferred (as in retropropagation or with support vector machines), but for applications as the control, in which input/output couples cannot be easily provided, these approaches fail and the genetic algorithms (as well as simulated annealing, which can be seen as a typical case) seem to be a natural solution.

Local optimizations can be made on the elements of a given generation, e.g. for a network of neurons, when one adapts the weights by retropropagation, and that one transmits these weights to the descendants. Such approaches make it possible in particular to use controllers' symbolic systems, 
called evolutionary programming, or neuronal controllers. The genetic algorithms can be used to optimize the weights but also architecture, by using genetic codes for architectures.

Simulated annealing is the case where one has only one element per generation; one adds noise to the fitness function (less and less vs. time) so as to have sometimes occasionally falls of fitness (in order to avoid local minima) to produce better elements than those of the former generations.

New papers are here introduced to improve our knowledge in terms of genetic algorithms applied to reservoirs.

Kaydani et al. (2014) introduced a multi-gene genetic programming (MGGP) algorithm to estimate permeability in heterogeneous oil reservoirs. The advantage of MGGP approaches is their ability to generate prediction equations without a prior for any relationship. Hence an equation for permeability prediction using MGGP was generated, based on well log and core experimental data from one Iranian oil reservoir. Comparison of the results shows that the MGGP is able to predict permeability effectively, with a good estimation despite high non-linearity relationship between petrophysical parameters. The MGGP model was then compared statistically to ANN, adaptive neuro-fuzzy inference system (ANFIS) and genetic programming (GP) models. ANN and ANFIS suffer from structure dependency, which affects their output results. They may sometimes be trapped in the local minimum during their training phase. MGGP model is formed of a number of relatively simple, fixed depth sub-models, less complex than the GP model. Hence, MGGP is able to generate compact models with close to experimental well log data and best results with low computational time compared to the other methods.

Modelling simulation of hydrocarbon reservoirs, operation and design of surface facilities, determination of inflow performance, estimation of oil and gas in place, and analysis of well testing and material balance data greatly depend on the fluid PVT properties like density and bubble point pressure (BPP). They are useful to make a proper plan for reservoir development. An effective and quick correlation was used by Ahmadi et al. (2014b) to accurately calculate the BPP of crude oil samples as a function of temperature, oil composition, molecular weight of $\mathrm{C}_{7_{+}}$, and specific gravity of $\mathrm{C}_{7+}$. In fact, adequate knowledge of reservoir fluid characteristics (e.g. BPP) plays a crucial role while conducting modelling/simulation of production processes in petroleum reservoirs. To obtain proper correlations for prediction of BPP of reservoir fluids, they applied, through an extensive statistical method, a novel ANN technique in the form of gene expression programming (GEP). The appropriate correlation has been obtained through the experimental/real data used for training and testing phases. The conventional predictive methods to estimate BPP as a function of independent parameters with the same datasets were also addressed. Comparing both the outputs obtained from the previous models with the BPP values predicted by the GEP technique, show that GEP approach exhibits much higher accuracy and lower uncertainty on the basis of statistical analysis in terms of coefficient determination $\left(\mathrm{R}^{2}\right)$ and MSE. 
Finally, in their documented paper, Aifa et al. (2014b) introduced a hybrid neuro fuzzy (NF) model based on the use of petrophysical data from well logs to predict porosity and permeability at depth. This petrophysical-based method has been applied to four wells in a shaly sand reservoir of Triassic Formation in Hassi R'Mel gas condensate field, Algeria. The well logs are analyzed for each well, then the results are correlated with core data information to produce reliable estimates between parameters. Fuzzy logic (FL) improves the generalization ability of a neural network (NN) dealing with implicit knowledge, the neural system by providing a more reliable output when extrapolation is needed beyond the limits of training data. In hybrid systems (NF), the combination of both can show the advantages of fuzzy systems, where information is treated explicitly with that of neural networks. This modelling approach, using first order Sugeno model, requires no previous hypothesis based on the complexity of the physical or experimental reservoirs or the building of a reasonable model from a specific set of measured data. The authors noticed that the use of NN and NF with FL, for prediction of porosity and permeability in a sandstone reservoir show low RMSE values of 0.1575 and 0.4556 and high correlation coefficients values of 0.9879 and 0.9689 for porosity and permeability, respectively, indicating that the NF method is the best tool for prediction compared to neural network.

\section{Conclusion}

We may notice that some of the problems regarding the applicability of ANN to reservoirs are related to their regularization, which seems to be a key point to obtain a "good" ANN. As it is difficult to get the right number of hidden layers, two solutions can be explored for a better generalization and regularization of ANN: (a) arrest in the training of the system before convergence, (b) moderating the weights. Such regularization can fit answer surfaces and has a good capacity for generalization. But in practice, several techniques of regularization are combined: (i) a "reasonable" number of hidden neurons according to the complexity of the answer surface and the number of examples available, reduced weight number, fast calculations, (ii) a base of formed validation by independent examples to ensure a good statistical representativeness of the network, but if data are lacking, cross validation is needed, (iii) a moderation of the weights to limit the effects of the exceeding parameters such as the parameter of weighting of the weights chosen according to accurate criteria expected from the network, for example target repeatability of output measurements. Different artificial intelligence techniques were applied and sometimes efficiently adapted to specific cases in the aforementioned papers. Yet, much work remains to be done, since complex heterogeneous reservoirs are being explored and the challenge is always to find numerical and simple low-cost practical solutions that are less timeconsuming.

\section{Acknowledgements}

This special volume was made possible thanks to the speedy participation of the contributors. I gratefully acknowledge the main authors for the submission of their papers, for their quick answers 
during the short delay to enable publication as quickly as possible. I also wish to thank all the colleagues who acted as reviewers, the editor-in-chief, and the journal managers.

\section{References}

Adeniran, A., Elshafei, M., Hamada, G., 2010. Artificial Intelligence Techniques in Reservoir Characterization: Functional networks softsensor for formation porosity and water saturation in oil Wells. ISBN: 36392442659783639244267 , VDM Verlag, Saarbrücken, Germany.

Ahmadi, M.A., Ebadi, M., 2014. Connectionist Model Predicts Porosity and Permeability of Petroleum Reservoirs by Means of Petro-Physical Logs: Application of Artificial Intelligence. Journal of Petroleum Science and Engineering, this issue.

Ahmadi, M.A., Ebadi, M., Yazdanpanah, A., 2014a. Robust intelligent tool for estimating dew point pressure in retrograded condensate gas reservoirs: Application of particles warm optimisation. Journal of Petroleum Science and Engineering, this issue.

Ahmadi, M.A., Zendehboudi, S., James, L., Elkamel, A., Dusseault, M., Shafiei, A., Chatsiz, I., Lohi, A., 2014b. New Tools to determine Bubble Point Pressure of Crude Oils: Experimental and Modeling Study. Journal of Petroleum Science and Engineering, this issue.

Aïfa, T., Ali Zerrouki, A., Baddari, K., Géraud, Y., 2014a. Magnetic susceptibility and its relation with fractures and petrophysical parameters in the tight sand oil reservoir of Hamra quartzites, southwest of the Hassi Messaoud oilfield, Algeria. Journal of Petroleum Science and Engineering, this issue.

Aïfa, T., Baouche, R., Baddari, K., 2014b. Neuro fuzzy system to predict permeability and porosity from well log data: a case study of Hassi R'Mel gas field, Algeria. Journal of Petroleum Science and Engineering, this issue.

Aminzadeh, F., de Groot, P., 2006. Neural Networks and Other Soft Computing Techniques with Applications in the Oil Industry. ISBN: 90-73781-50-7, EAGE Publ., 161p.

Ashton Acton, Q., 2013. Advances in Machine Learning Research and Application. ISBN: 978-1-48168319-7, ScholarlyEditions, Atlanta, GA.

Chaki, S., Verma, A.K., Routray, A., Mohanty, W.K., Jenamani, M., 2014. Well tops guided prediction of reservoir properties using modular neural network concept: Acase study from western onshore, India. Journal of Petroleum Science and Engineering, this issue.

Costa, L.A.N., Maschio, C., Schiozer, D.J., 2014. Application of artificial neural networks in a history matching process. Journal of Petroleum Science and Engineering, this issue.

Enab, K.,Ertekin, T., 2014. Artificial neural network based design for dual lateral well applications. Journal of Petroleum Science and Engineering, this issue.

Foroud, T., Seifi, A., AminShahidi, B., 2014. Assisted History Matching using Artificial Neural Network based Global Optimization Method- Applications to Brugge Field and a Fractured Iranian Reservoir. Journal of Petroleum Science and Engineering, this issue. 
Ghaffarian, N., Eslamloueyan, R., Vaferi, B., 2014b. Model identification for gas condensate reservoirs by using ANN method based on well test data. Journal of Petroleum Science and Engineering, this issue.

Graupe, D., 2007. Principles of Artificial Neural Networks. Advanced Series on Circuits and Systems. $2^{\text {nd }}$ Ed., vol.6, World Scientific Publishing Co. Pte. Ltd., 303p.

Jerry Lucia, F., 2007. Carbonate Reservoir Characterization. An integrated Approach. $2^{\text {nd }}$ Ed., ISBN: 978-3-540-72740-8 Springer, Berlin, 336p.

Kaydani, H., Mohebbi, A., Eftekhari, M., 2014. Permeability estimation in heterogeneous oilr eservoirs by multi-gene genetic programming algorithm. Journal of Petroleum Science and Engineering, this issue.

Khamehchi, E., Kivi, R.I., Akbari, M., 2014. A novel approach to sand production prediction using artificial intelligence. Journal of Petroleum Science and Engineering, this issue.

Maschio, C., Schiozer, D.J., 2014. Bayesian history matching using artificial neural network and Markov Chain Monte Carlo. Journal of Petroleum Science and Engineering, this issue.

Masoudi, P., Arbab, B., Mohammadrezaei, H., 2014a. Net pay determination by artificial neural network: Case study on Iranian offshore oil fields. Journal of Petroleum Science and Engineering, this issue.

Masoudi, P., Arbab, B., Mohammadrezaei, H., 2014b. Net pay determination by Dempster rule of combination: Case study on Iranian offshore oil fields. Journal of Petroleum Science and Engineering, this issue.

Mosobalaje, O.O., Orodu, O.D., Tiab, D., 2014. New Techniques for Estimating Properties of Saturated Reservoirs, Using Readily-Available Rate Decline Data. Journal of Petroleum Science and Engineering, this issue.

Nikravesh, M., Aminzadeh, F., Zadeh, L.A., (eds.), 2003. Soft Computing and Intelligent Data Analysis in Oil Exploration. ISBN: 0-444-50685-3, $1^{\text {st }}$ Ed. Elsevier, 723p.

Rabbani, A., Jamshidi, S., Salehi, S., 2014. An automated simple algorithm for realistic pore network extraction from micro-tomography Images. Journal of Petroleum Science and Engineering, this issue.

Sandham, W., Leggett, M., (eds.), 2003. Geophysical Applications of Artificial Neural Networks and Fuzzy Logic. Kluwer Acad. Publ., ISBN: 1-4020-1729-4, Dordrecht, The Netherlands,

Sheremetov, L., Cosultchi, A., Martínez-Muñoz, J., Gonzalez-Sánchez, A., Jiménez-Aquino, M.A., 2014. Data-driven forecasting of naturally fractured reservoirs based on nonlinear qutoregressive neural networks with exogenous input. Journal of Petroleum Science and Engineering, this issue.

Shi, G., 2014. Data Mining and Knowledge Discovery for Geoscientists. Petroleum Industry Press, Elsevier.

Shokooh Saljooghi, B., Hezarkhani, A., 2014. Comparison of WAVENET and ANN for predicting the porosity obtained from well log data. Journal of Petroleum Science and Engineering, this issue. 
Yanfang, W., Salehi, S., 2014. Refracture Candidate Selection Using Hybrid Simulation with Neural Network and Data Analysis Techniques. Journal of Petroleum Science and Engineering, this issue.

Tahar Aïfa

Géosciences-Rennes, CNRS UMR6118, Université de Rennes 1, Bat.15, Campus de Beaulieu, 35042, Rennes cedex, France E-mail : tahar.aifa@univ-rennes1.fr 